2014

\title{
Parts of the Whole: Strategies for the Spread of Quantitative Literacy: What Models Can Tell Us
}

Dorothy Wallace

Dartmouth College, dorothy.wallace@dartmouth.edu

Follow this and additional works at: https://digitalcommons.usf.edu/numeracy

Part of the Community College Leadership Commons, Educational Leadership Commons, Higher Education Commons, and the Science and Mathematics Education Commons

\section{Recommended Citation}

Wallace, Dorothy. "Parts of the Whole: Strategies for the Spread of Quantitative Literacy: What Models Can Tell Us." Numeracy 7, Iss. 2 (2014): Article 7. DOI: http://dx.doi.org/10.5038/1936-4660.7.2.7 


\title{
Parts of the Whole: Strategies for the Spread of Quantitative Literacy: What Models Can Tell Us
}

\author{
Abstract \\ Two conceptual frameworks, one from graph theory and one from dynamical systems, have been offered \\ as explanations for complex phenomena in biology and also as possible models for the spread of ideas. \\ The two models are based on different assumptions and thus predict quite different outcomes for the \\ fate of either biological species or ideas. We argue that, depending on the culture in which they exist, one \\ can identify which model is more likely to reflect the survival of two competing ideas. Based on this \\ argument we suggest how two strategies for embedding and normalizing quantitative literacy in a given \\ institution are likely to succeed or fail.

\section{Keywords} \\ ducation reform, memetics, quantitative literacy, quantitative reasoning, numeracy \\ Creative Commons License \\ (c) (i) (9) \\ This work is licensed under a Creative Commons Attribution-Noncommercial 4.0 License

\section{Cover Page Footnote} \\ Dorothy Wallace is a professor of mathematics at Dartmouth. She was 2000 New Hampshire CASE \\ Professor of the Year, and the lead PI of the seminal NSF project, Mathematics Across the Curriculum. \\ She recently finished a text in mathematical biology for first-year students, "Situated Complexity." She was \\ a charter board member of the National Numeracy Network and is now co-editor of this journal.
}




\section{Parts Of The Whole A Column by $\mathrm{D}$. Wallace}

The problem of how best to improve the numeracy of a society is a thorny one, embracing the learning process of a single student but rising in scale to include the management and alteration of an entire system of education. With the issue of quantitative literacy always in mind, this column considers various aspects of the systemic workings of education, the forces acting on classrooms, teachers and students, and mechanisms of both stasis and change.

\section{Strategies for the Spread of Quantitative Literacy: What Models Can Tell Us}

Numeracy, defined both in conjunction with and as an alternative to traditional mathematics curriculum and instruction, is still struggling to find its place in the constellation of requirements at the high school and college level. Will it persist as a course requirement, viewed by students as just another obstacle to graduation? Will it be become the charge of a mathematics department and compete for the attention of both students and faculty with familiar, valued courses? Will it be offered by a separate "center" or other educational body, with the danger that it is marginalized by the institution? Will it become a concept so successful that it is internalized in a wide variety of courses, but still avoidable by students determined to avoid all things generally mathematical? All of these are possible and at some level would be considered a wild success compared to simply ignoring the need to produce a quantitatively literate population. The reason any of these outcomes would be considered successful is that each of them achieves a kind of stability for numeracy within the educational system.

Those inclined to resist change in education think of new approaches as "fads" which will, with luck, go the way of the pet rock. Those promoting change hope that they will stick, becoming embedded in the culture in a stable way. To consider the issue from a memetics point of view, we will clarify what exactly is meant by "stability" in the sense of both mathematics and ecology.

\section{What Does Stability Look Like?}

To get a better picture of the behavior of possible histories in a complex system it is useful to visualize it the way a mathematician would. Each quantity tracked, or species in an ecology, has a starting population. Let us say there are a hundred species in the system. Then the starting place is a point in hundred-dimensional space whose coordinates are the hundred different starting populations. This space of all possible configurations of the system is called its "state space." As 
the equations that govern the system go into play, the populations of all these species begin to change, giving a curve passing through the state space. The state space in turn is filled up with these trajectories, each of which represents the fate of the original configuration. The curves, taken together, are called a "flow." A flow in state space behaves somewhat like a flowing fluid, except that it can do much more complicated things because it doesn't have to obey physics and because it has more dimensions than a physical fluid. It can do all the things a real fluid does-make eddies, have fixed points that don't move at all, spiral inward toward a region (which is called an attractor) or outward away from itand then some.

So, a closed trajectory, say a loop or cycle within the system, will be unstable if nearby trajectories diverge from it. Models abound with unstable loops or closed surfaces. In the world of "memes," the word coined to describe units of thought, such a situation would represent a set of ideas interacting with each other in a superficially persistent way but which will ultimately degenerate into a completely different configuration when any new idea or piece of data perturbs them into a slightly different relationship with each other. By contrast, a stable configuration can persist in the face of small perturbations. Stability in this sense is usually achieved by the presence of an attractor. Because all configurations represented in the region around the stable attractor (the basin of attraction) tend to the same final configuration, small perturbations do not affect the final result much at all.

An unstable configuration in a dynamical system is nearly impossible to observe, either in the model or in the real situation described by it. If the brute force of theoretical mathematics cannot identify such a configuration, then a researcher is reduced to searching for it with a computer. Because even the best computer makes tiny approximations when it computes (called round-off error), even if you were lucky enough to start out on the unstable loop, you would eventually depart from it and head for the nearest stable configuration. Because nature also has small random events, you would never see an unstable configuration in a real system either.

From a mathematical standpoint, what stability means is not at all the same thing as the common meaning of the term. When we read in the paper that "the situation in Palestine is stable," the writer means that two groups of humans are momentarily living in relative peace. When an ecologist says a system is stable it does not mean that species are living together in peace, nor even in stasis. Populations will continue to rise and fall: competitive species will continue to compete; predators will continue to eat prey. What stable means in this case is that the collections of species and their patterns of interaction, no matter how complicated or unpredictable in the long term, will continue to exist and function indefinitely in the face of small random perturbations.

In the world of ideas, the mathematical notion of stability should not be confused with peaceful coexistence. In fact, the stability of a set of ideas may 
require opposition and argument. One could argue, for example, that centuries of rabbinical dispute over Judaic law not only made the law richer and more complicated, but also made the initial collection of ideas more stable. Mathematical biology has given a new way to argue about the principles of ecology that has the effect of cementing those principles into a more or less permanent body of knowledge. The dynamic at work here is dialectic-as described by Marx, thesis requiring antithesis for its very survival. One idea may dominate the other for a long time, only to suddenly shift in importance. Because people are the vectors for ideas and, because people are emotional, volatile, and even violent, human strife and misery are a natural consequence of the interaction of ideas, even collections of ideas that are inherently stable.

So let us be clear about this mathematical and biological concept of stability. A stable configuration of ideas can include argument, strife, and changing levels of importance for the various ideas in the system. Stability, in the mathematical sense, guarantees merely the continuation of the existence of these ideas in play intellectually, as well as the continuation of their relationship to one another, but not their peaceful coexistence.

The language of dynamical systems and information theory doesn't take into account what ideas do best: change, adapt, and interact to produce new variations on old ideas. In the language of dynamical systems, the only pathway to a new configuration is through some perturbation that knocks the system into a new basin of attraction: same ideas, some now extinct, different visible patterns of ascendance. Real ideas evolve, and synthesize new ideas, most of which suffer the fate of the pet rock. The point of view taken in this essay is limited, but even so sheds some light on how the fate of a new idea may play itself out.

\section{Is it Possible for Diversity to be Stable in the World of Ideas?}

Let us start with Bosnia. For a large portion of the twentieth century, Bosnia was the home to thriving populations of both Christians and Muslims, living and working side by side. Two entire religious systems complete with some contradictory doctrine lived in the population of a relatively small geographical area. People from these different traditions sometimes intermarried, which shows that even within a single individual there could be a high tolerance for contradictory ideas, a requirement if not a sufficiency for intellectual diversity. Evidently the balance was precarious because, as we know, the tides of doctrine turned quite abruptly into political action purging the Muslims from their homes, to the great surprise of many who had thought that the situation looked stable. Yet, on a global scale the situation still looks stable. Both sets of religious ideas coexist for the most part without open conflict. It would be hard to argue in favor of such apparent stability on the basis of the actual doctrines of the two systems. A larger principle must be at work. 
Ecologists have grappled with the same sort of phenomenon in the natural world. If two species are in direct competition for exactly the same resources, then, in the absence of other forces, one will often out-compete the other, driving it to extinction. This is true both for the mathematical model one can construct and also for many ecological systems we can observe. In Lake Victoria, the introduction of Nile perch drove many species of cichlid to extinction. Millefoil is considered a noxious weed because it out-competes the native plants in lakes throughout the United States where it is accidentally introduced. Eradication of a whole population and extinction of species are fairly normal events. Yet we also see systems of incredible diversity, such as tropical forests, where even cataloguing the species is an impossibly large task. For a good description of how ecologists think about this pair of seeming contradictions, the reader is referred to the excellent discussion in "Why Big Fierce Animals Are Rare" by Paul Colinvaux. ${ }^{1} \quad$ We will summarize that discussion although with a more mathematical point of view.

There are two different mathematical paradigms for describing a complex ecosystem. One viewpoint comes from graph theory. Information theorists use this kind of approach when studying things like telephone networks. A graph consists of vertices and edges. To model an ecosystem we would assign each species to a vertex and connect two vertices if the species interact along the food chain. Energy flows through the network. Grass would be connected to rabbits; rabbits would be connected to foxes and so on. This approach can also be adjusted to take into account the size of the population of the species. In the case of telephone networks, the nodes represent switches and the edges might represent actual wires. Information flows through the network. There are various measures, in particular the Shannon-Wiener measure, which attempt to capture the complexity of such a graph. It turns out that the more complex the graph is the more dependably it can move information around. The lesson from information theory is that complexity assures stability in this sense.

Here is an example of a mathematical model where the answer that comes out of the model resembles a phenomenon that ecologists believed in already: the stability of complex systems. On the other hand, the interaction between species is not like that of a switch. When a switch sends information towards another switch, the information has moved but the network has not changed. When a fox meets a rabbit, the rabbit population goes down and the nature of the network is different from before. The mechanism of animal interaction is not mirrored very well in the graph-theoretic model. At the time that ecologists promoted the results of information theory as a description of ecosystems, computers were not very advanced. Capturing the subtle nature of the interaction between species requires the use of differential equations, one equation for each vertex of the graph that the

${ }^{1}$ Colinvaux, Paul A. Why big fierce animals are rare: an ecologist's perspective. Princeton University Press, 1979. 
information theorist uses. Solving so many equations at a time is impossible except through numerical techniques beyond the scope of early computers. As computers improved, the ecologists gained a model that more accurately reflected the mechanism of species interaction.

One way to investigate the behavior of a complex system driven by a collection of differential equations is to pick a starting point, a set of initial conditions, for the system. One then uses numerical methods and a good machine to estimate subsequent states of the system. For an ecosystem, picking a starting point means deciding on an initial population for every species in the system. So the ecologists set up their equations, picked some initial conditions, and watched what happened. They were surprised at the result. For certain initial settings the system ran more or less as expected, but for many choices of initial conditions, the populations of species changed in such a way that many species went extinct. The system went from more complex to less complex, absolutely on its own. Eventually it might reach a simpler system with fewer species that was stable in the sense that it could run forever with no further extinctions. The lesson of differential equations is that complexity does not guarantee stability at all for the kinds of relationships governing ecosystems. Simple ecosystems are more likely to be stable.

It helps to visualize a state space as divided into regions where different kinds of behavior predominate. In some regions the trajectories may spiral rapidly toward a fixed point or closed path. We divide the state space into regions depending on these "attracting sets" because the attractors describe the eventual fate of all the starting places within the region of attraction. So what we learn from the computer models of a complex ecosystem is that the region of stability for some attractor, which includes nonzero populations of all the species in the model, is not the whole space. There are other attractors lying on hyperplanes where one or many species are extinct, and if the system begins inside the region of attraction for that attractor, then it will eventually lose the corresponding species.

Now we can see a little better why some ecosystems are more complex than others. Given good conditions under which many species can arise and coexist, an ecosystem may prosper and include many species. But if the system is perturbed by climate change, the introduction of some new species, or some other external factor, then the state of the system might get suddenly pushed into a different region of attraction. We would then see the sudden disappearance of any species not present in the attractor. This general mathematical gestalt would lead us to expect to see simpler ecosystems in places where the climate was inhospitable and prone to disaster, such as near the poles, and complex systems in regions where the climate was mild and conditions didn't vary wildly, such as in the tropics. Such a pattern does in fact hold. So, although most of the attracting sets may lie on "hyperplanes of extinction," complexity can exist if the system is never perturbed. 
Ecologists have chosen dynamical systems over information theory as the more believable model. But we have to return to first principles and try to decide which of these two models is a better approximation of the exchange of ideas, the one coming from information theory or the one coming from differential equations? Colinvaux argues that information theory is a poor model of ecosystems because the predator-prey interaction is one in which one party seeks to disrupt the flow of energy through the system. The prey does not wish to be eaten. This particularity of behavior, however, is not present in the equations governing the interaction. Stare at them as long as you will, you will not be able to read from them any resistant behavior of the organism. The equation looks the same whether the prey is a mobile animal or a stationary grass. Colinvaux's reasoning alone does not suffice as an explanation of the different outcomes of these two mathematical models.

The main difference in the models is in what happens when the system is perturbed. In the information-theory model, if a vertex is removed the information finds other pathways when it can. In the differential-equations model, if a species is removed, all of the relationships are changed and many more species may disappear. The energy does find other pathways, but at the cost of many nodes. A perturbation in the graph-theory model results in a different distribution of the energy flow, but not in a major restructuring of the configuration of the graph. It is this property that allows for "stability" in the sense of continued information flow to all parts of the network.

If we think about how ideas move from one person to another and interact with other ideas, we will see immediately that it is a rare event for one idea to obliterate another in the same sense that a predator consumes its prey. Ideas move throughout a culture by connecting with other ideas, but that connection is fluid and multidirectional. There are many different justifications that can be offered for a particular idea. The "success" of an idea depends more on its ability to connect with accepted ideas and move fluidly from context to context. Therefore ideas usually behave far more like the flow of information through a network than like individual species interacting by consuming each other. We would therefore expect a multiplicity of ideas to be the norm. We would expect even relatively out-of-date and unimportant ideas to persist in a culture, occupying small niches and travelling on the less-worn paths through the network. Diversity should improve stability, in the sense of persistence of ideas, in the absence of other factors.

In situations where the mechanisms by which ideas interact can justifiably be described by differential equations, they are then subject to the same sort of phase-portrait analysis we described earlier. Although the complex situation may be stable, it is not the only stable configuration possible. Stress could move the system to a different configuration, probably simpler than the previous one.

What, then, of Bosnia? Our discussion would suggest that in times of peace and prosperity the coexistence of Christian and Moslem thought in a population 
would be normative. The religious people of Bosnia were put under considerable stress by the politics of surrounding lands, to the point of having to develop an artificial intolerance of each other's belief systems in order to survive an external physical threat. Under stress, competition arose between two belief systems. Where there is competition there is a model available from differential equations to describe it. Under many conditions the model predicts only one surviving competitor.

This interpretation fits well with what we know about complex systems from both mathematical viewpoints. On a small scale, in a stressful situation that puts the two beliefs into immediate competition, we see one triumph over another. On a larger scale, the coexistence of Moslem and Christian thought appears quite stable, with many examples of communities where mutual acceptance is the norm. Together, history and mathematics both give us some cause to rejoice in the longterm possibility of tolerance.

\section{Numeracy as a Stable Meme}

Our discussion suggests some lessons for the quantitative literacy movement. Two examples demonstrate how these principles can inform our choice of strategies in promoting numeracy and also our expectations for those strategies.

Embedding quantitative reasoning in many courses across the curriculum is a flexible strategy that has the advantage of keeping it out of competition for all the resources it takes to sustain courses in general. It links numeracy to many other ideas. In the language of information theory, quantitative reasoning would be a node of high degree, promoting stability. Instructors from many disciplines would value the skills and insight numeracy offers their students and would come to expect it. There would still be paths through the curriculum that would allow students to avoid quantitative tasks in this scenario. It would also be difficult to measure success of an institution or program, as it would be unclear at what point to take measurements or against what benchmark to test. The very diversity of ideas that would aid in stability would also thwart measurement.

On the other hand, creating a separate set of requirements, courses and tests can at most capture only a small slice of what it means to be numerate. The advantage of such a strategy is that it becomes possible to know if a particular student has mastered a particular body of knowledge and also possible to measure whether an institution or program has succeeded in improving the skills and knowledge of students in general, as benchmarks are easier to identify. One disadvantage is that an obvious and large allocation of resources is necessary to follow this strategy, resources that could be used in many other ways. Any special program, course or center is now in competition for these resources, being delineated as "other," distinct from existing courses and disciplines. The language of dynamical systems is a better description of this situation, as many players vie for funding and also for the attention of students, both of which can be 
scarce resources. Based on the discussion above, we would expect this strategy to fail fairly often, just as species are often driven extinct when they compete. We would expect it to succeed particularly well when resources are rich, competitors few, and when there are few shocks to the system, economic or otherwise. Finally, we would expect that, even when these courses and centers are long lived, stability would take the form of continued competition, argument, and defense of such a use of resources.

The need for a quantitatively literate population is not ephemeral. The consequences are major and unpleasant in perpetuating a nation of citizens who cannot manage their money, make quantitatively informed decisions at home or at the polls, and who cannot supply from their numbers the scientists, inventors and quantitatively skilled workers of the future. It is critical that numeracy not be just another educational fad. By understanding how ideas come to be familiar, accepted, and deeply woven into a culture, perhaps we will be able to create strategies that position quantitative literacy where it belongs in education: alongside reading and writing as an expected skill of every educated person. 